\section{Die ärztliche Begutachtung}

— Seit 1982 erscheint dieses Standardwerk zur ärztlichen Begutachtung. In der aktuellen 8. Auflage wird traditionell neben den allgemeinen Grundlagen sehr speziell auf die Funktionsprüfungen und die Diagnostik der verschiedenen Fachgebiete der Medizin als Grundlage für die Begutachtung eingegangen. Somit eignet sich dieses Werk nicht nur für die Gutachter aller medizinischen Bereiche hervorragend, sondern auch und vor allem für Hauptgutachter, die den Gesamtschaden unter Gewichtung mehrerer Gutachten unterschiedlicher Fachgebiete feststellen müssen. Das Werk beeindruckt dabei durch eine sachliche Darstellung der Inhalte unter Verzicht auf den übermäßigen Einsatz von Farben im Layout, die in modernen Lehrbüchern teilweise inflationär gebraucht werden und dabei den Blick auf das Wesentliche, das sie eigentlich hervorheben sollten, eher verstellen denn erleichtern. Aus diesen Gründen kann dieses Buch jedem medizinischen Gutachter sehr empfohlen werden.

PDDr. R. Gaulke

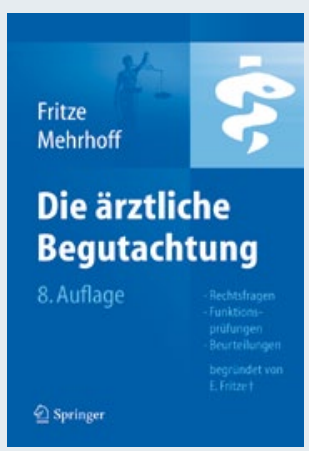

\section{Die arztliche Begutachtung}

Rechtsfragen, Funktionsprüfungen,

Beurteilungen. Fritz J., Mehrhoff F. (Hrsg.),

Springer Verlag; 8., vollst. überarb. u. akt.

Aufl. 2012; geb., 901 S., 90 Abb.;

Preis (inkl. MwSt.) 199,95 Euro;

ISBN 978-3-642-21080-8

\section{Der kapriziöse Ellenbogen}

— Das verletzte Ellbogengelenk will mit höchster Sorgfalt und genauester präoperativer Planung angegangen werden. Sei dies nicht der Fall, räche sich dieses "kapriziöse" Gelenk" - zu Ungunsten des Patienten, mahnen die Autoren im Vorwort zu ihrem reich und eindrucksvoll bebilderten Buch „Der kapriziöse Ellenbogen“. An 51 Fällen aus der Praxis beschreiben erfahrene Ellenbogen-Chirurgen unterschiedlichste Erkrankungen und Verletzungen des Ellenbogengelenks mit Unfallhergang, Versorgung und Follow-up. Anhand der Kasuistiken werden Indikationen und Stellenwert der Ellbogenarthroskopie ebenso diskutiert wie die Langzeitresultate der Ellbogen-Totalprothese oder die Implantation einer Radiusköpfchenprothese und viele weitere Besonderheiten. Nach der Lektüre des Buches versteht der faszinierte Leser, warum das Autorenteam das Ellenbogengelenk „kapriziös“ nennt.

Dr. Brigitta Schneider

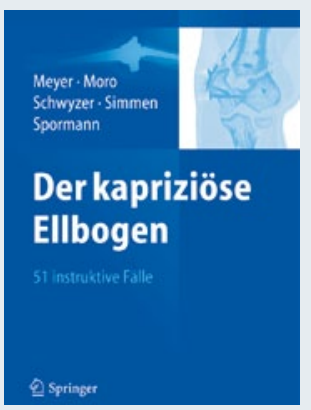

Der kapriziöse Ellbogen - 51 instruktive Fälle

Meyer, R.-P.; Moro, F.; Schwyzer, H.-K.; Simmen, B.R.; Spormann, C. (Hrsg.); Springer Verlag 2012; geb.; 310 S., 600 Abb.; Ladenpreis (inkl. MwSt.) 69,95 €; ISBN 978-3-642-25880-0; auch als PDF-Download verfügbar (ohne DRM, mit Wasserzeichen)

\title{
FBL Functional Kinetics. Ballübungen
}

— Übungen mit dem Gymnastikball sind wichtige Bausteine im Therapiekonzept FBL Functional Kinetics. Dieser Baustein des FBL-Konzepts lässt sich in Prävention und Therapie gleichermaßen wirksam einsetzen und mit anderen Verfahren kombinieren. Der Ball fördert Fertigkeiten, die wesentlich für ein optimales Bewegungsverhalten sind, nämlich Reaktionsfähigkeit, dynamische Stabilisation und ökonomische Aktivität. Das Lehr- und Übungsbuch zeigt ganz praktisch, wie die Ballübungen funktionieren und wie man die Patienten dazu anleitet. In der 5. Auflage sind die Übungen noch leichter verständlich und übersichtlicher dargestellt. Zudem illustrieren erstmals detailreiche Farbfotos höchst anschaulich die Bewegungsabläufe.

Eine besonders wertvolle Neuerung ist die DVD, die dem Buch nun erstmals beiliegt: _ Alle geschilderten Ballübungen Ballübungen sind in Videofilmen zu besichtigen.

_ Auf der DVD enthaltene bebilderte Schulungsblätter mit leicht verständlicher Anleitung, Tipps zum Üben usw. kann man ausdrucken und den Patienten zum häuslichen Üben mitgeben.

Dr. Brigitta Schneider

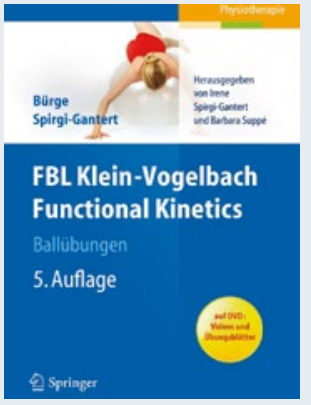

FBL Functional Kinetics. Ballübungen Bürge Elisabeth, Spirgi-Gantert Irene Spirgi-Gantert Irene; Suppé Barbara (Hrsg.) 5., vollst. überarb. Aufl.; Springer Verlag 2013; Verfügbare Formate: eBook: direkt als PDF-Download verfügbar (ohne DRM, mit Wasserzeichen); Preis inkl. MwSt. 35,99€;

ISBN 978-3-642-22070-8 195 S.; mit DVD; Preis inkl. MwSt. 39,95 €; ISBN 978-3-642-22069-2 